PROCEEDINGS OF THE

AMERICAN MATHEMATICAL SOCIETY

Volume 35, Number 2, October 1972

\title{
ON THE EQUIVALENCE OF THREE LOCAL THEOREM TECHNIQUES
}

\author{
K. K. HICKIN AND J. M. PLOTKIN ${ }^{1}$
}

\begin{abstract}
A result formulated by $P$. Hall which is used in obtaining local theorems in group theory is shown to be equivalent to the projection set theorem of Kuroš and to the existence of Malcev vectors for local systems.
\end{abstract}

0. Introduction. It was Malcev [3] who first stated and proved local theorems in group theory. To explain the term "local theorem" we need some preliminary definitions.

Let $S$ be a set. A local system $L$ on $S$ is a collection of subsets of $S$ such that for each finite subset $\left\{x_{1}, \cdots, x_{n}\right\} \subseteq S$ there is an $H \in L$ with $\left\{x_{1}, \cdots, x_{n}\right\} \subseteq H$. It follows immediately if $L_{1}$ is a local system on $S$ and $L_{2}$ is a collection of subsets of $S$ with $L_{1} \subseteq L_{2}$ then $L_{2}$ is a local system on $S$.

Let $\Sigma$ be a class of groups. We say that a group $G \in L(\Sigma)$ if there is a local system on $G$ consisting of subgroups in $\Sigma$. We always have $\Sigma \subseteq L(\Sigma)$. A local theorem for $\Sigma$ asserts that $\Sigma=L(\Sigma)$. For examples of such classes see Kuroš [2] or D. J. S. Robinson [6].

The original methods of Malcev involved the use of logical techniques. However, the needed technical theorem can be stated without any reference to first order logic. One such formulation due to P. Hall appears with a proof in D. J. S. Robinson [6]. Another version due to A. Robinson and very similar to Hall's appears in [5]. We now state a variant of Hall's theorem.

THEOREM H. Let $L$ be a local system on $S, F$ a set, $n$ a positive integer. Suppose that for each $H \in L$ there is a function $f_{H}: H^{n} \rightarrow F$ and $\left\{f_{H}(x) \mid H \in L\right\}$ is finite for each $x \in S^{n}$. Then there is a function $f: S^{n} \rightarrow F$ such that for any finite subset $K \subseteq S^{n}$ there is an $H \in L$ with $K \subseteq H^{n}$ and $f|K=f| H$.

Received by the editors December 1, 1971 and, in revised form, February 7, 1972. AMS 1970 subject classifications. Primary $20 \mathrm{E} 25$.

Key words and phrases. Local system, inverse limit system, Malcev vector, Boolean prime ideal theorem.

1 The second author wishes to thank K. K. Hickin and R. E. Phillips for introducing him to local theorems. 
Remarks. In P. Hall's original formulation $F$ is a finite set. One can derive Theorem $\mathrm{H}$ from Hall's theorem.

In $\S 1$ we apply Theorem $H$ to inverse limits systems to obtain the projection set theorem of Kuroš. In $\S 2$ we use Kuroš's theorem to prove the existence of Malcev vectors which in turn yields a proof of Theorem $\mathrm{H}$. We discuss the strength of these theorems in $\$ 3$.

1. Inverse limit systems. Let $\langle I, \leqq\rangle$ be an upper directed partially ordered set. A collection of sets $\left\{A_{i} \mid i \in I\right\}$ and functions $\left\{\varphi_{i j} \mid i, j \in I, i \leqq j\right\}$ is called an inverse limit system if

(1) $\varphi_{i j}: A_{j} \rightarrow A_{i}$;

(2) $\varphi_{i i}$ is the identity on $A_{i}$; and

(3) $\varphi_{i k}=\varphi_{i j} \circ \varphi_{j k}, i \leqq j \leqq k$. The inverse limit $A^{\infty}$ of such a system is

$$
\left\{p \in \prod_{i \in I} A_{i} \mid \forall i, j \exists k i, j \leqq k \exists a \in A_{k} \varphi_{i k}(a)=p(i) \& \varphi_{j k}(a)=p(j)\right\} .
$$

Using Theorem $\mathrm{H}$ we now prove a form of Kuroš's projection set theorem [2, p. 167].

THEOREM K. If $\left\{A_{i}\right\},\left\{\varphi_{i j}\right\}$ is an inverse limit system with each $A_{i} \neq \varnothing$ and finite, then $A^{\infty} \neq \varnothing$.

Proof. Using the axiom of choice for families of finite sets form $\left\{a_{i} \mid i \in I, a_{i} \in A_{i}\right\}$. For each $j \in I$ let $H_{j}=\{i \mid i \leqq j\}$. Let $L=\left\{H_{j} \mid j \in I\right\} . L$ is a local system on $I$. For each $H_{i} \in L$ define $f_{H_{i}}: H_{i} \rightarrow \bigcup_{i \in I} A_{i}$ as follows: $f_{H_{i}}(j)=\varphi_{j i}\left(a_{i}\right)$. Since $\left\{f_{H_{i}}(j) \mid H_{i} \in L\right\} \subseteq A_{j}$ and $A_{j}$ is finite, we can apply Theorem $\mathrm{H}$ and obtain a function $f: I \rightarrow \bigcup_{i \in I} A_{i}$. We now prove $f \in A^{\infty}$. Clearly $f(i) \in A_{i}$. Let $K=\{i, j\}$. There is an $H_{k}, K \subseteq H_{k}$ and $f_{H_{k}}|K=f| K$. Hence $f(i)=\varphi_{i k}\left(a_{k}\right)$ and $f(j)=\varphi_{j k}\left(a_{k}\right)$. Therefore $f \in A^{\infty}$.

2. Finite partitions of a local system. Let $L$ be a local system on $S$. A finite partition $\sigma$ of $L$ is a set $\left\{L_{i}^{\sigma} \mid i \leqq n\right\}$ such that $L_{i}^{\sigma} \cap L_{j}^{\sigma}=\varnothing$ for $i \neq j$ and $\bigcup\left\{L_{i}^{\sigma} \mid i \leqq n\right\}=L$. The following is easily proven:

LEMMA 2.0. If $L$ is a local system on $S$ and $\left\{L_{i}^{\sigma} \mid i \leqq n\right\}$ is a partition of $L$, then there is an $i_{0} \leqq n$ with $L_{i_{0}}^{\sigma}$ a local system on $S$.

Definition. $\quad L$ is a local system. Let $P=\{\sigma \mid \sigma$ a finite partition of $L\}$. A Malcev vector for $L$ is a function $f$ on $P$ such that $f(\sigma)$ is an element of the partition $\sigma$, and for any $\left\{\sigma_{1}, \cdots, \sigma_{n}\right\} \subseteq P$ we have $\bigcap\left\{f\left(\sigma_{i}\right) \mid i \leqq n\right\}$ is a local system on $S$.

The following theorem on Malcev vectors appears in McLain [4]. Our proof is based on Theorem $\mathrm{K}$. 
THEOREM M. Let L be a local system on $S$. There is a Malcev vector for $L$.

Proof. Let $P=\{\sigma \mid \sigma$ a finite partition of $L\}$. We partially order $P: \sigma \leqq \tau$ iff $\tau$ refines $\sigma$, i.e., each element of $\tau$ is contained in a (unique) element of $\sigma$. $\langle P, \leqq\rangle$ is upper directed since any $\sigma_{1}, \sigma_{2}$ have a common refinement $\tau$. For each $\sigma \in P$ let $A_{\sigma}=\left\{L_{i}^{\sigma} \mid L_{i}^{\sigma}\right.$ is a local system on $\left.S\right\}$. $A_{\sigma}$ is clearly finite and $A_{\sigma} \neq \varnothing$ by Lemma 2.0. For $\sigma \leqq \tau$ define $\varphi_{\sigma \tau}: A_{\tau} \rightarrow A_{\sigma}$ by $\varphi_{\sigma \tau}\left(L_{i}^{\tau_{i}}\right)=$ the unique $L_{j}^{\sigma}$ such that $L_{i}^{\tau} \subseteq L_{j}^{\sigma}$. Clearly $\left\{A_{\sigma} \mid \sigma \in P\right\},\left\{\varphi_{\sigma \tau} \mid \sigma \leqq \tau ; \sigma, \tau \in P\right\}$ is an inverse limit system. By Theorem $\mathrm{K}$ there is an $f \in A^{\infty}, f: P \rightarrow \bigcup A_{\sigma}$, $f(\sigma) \in A_{\sigma}$. Given $\left\{\sigma_{1}, \cdots, \sigma_{n}\right\}$ since $f \in A^{\infty}$ it is not hard to prove that there is a $\tau, \tau \geqq \sigma_{i}$ each $i$ and a $L_{j}^{\tau} \in A_{\tau}$ such that $\varphi_{\sigma_{i} \tau}\left(L_{j}^{\tau}\right)=f\left(\sigma_{i}\right)$. But then $L_{j}^{\tau} \subseteq f\left(\sigma_{i}\right), i \leqq n$. Since $L_{j}^{r}$ is a local system, $\bigcap\left\{f\left(\sigma_{i}\right) \mid i \leqq n\right\}$ is a local system.

By a slight modification of a proof in $[6$, p. 132] we can show that Theorem $\mathrm{M} \Rightarrow$ Theorem $\mathrm{H}$.

INDICATION OF PROOF. We are assuming the hypotheses of Theorem $\mathrm{H}$. For $x \in S$ let $\left\{f_{H}(x) \mid H \in L\right\}=\left\{u_{1}, \cdots, u_{n}\right\}$. We define the following partition $\sigma_{x}$ of $L$.

$$
\begin{aligned}
L_{n+1}^{\sigma_{x}} & =\{H \in L \mid x \notin H\}, \\
L_{i}^{\sigma_{x}} & =\left\{H \in L \mid x \in H \& f_{H}(x)=u_{i}\right\}, \quad 1 \leqq i \leqq n .
\end{aligned}
$$

Note that $L_{n+1}^{\sigma_{x}}$ is not a local system. By Theorem $M$ we have a Malcev vector $g$ for $L$. Define $f: S \rightarrow F$ by $f(x)=u_{i}$ iff $g\left(\sigma_{x}\right)=L_{i}^{\sigma_{x}}$. One can check that $f$ has the desired property.

3. Final comments. In [6] Theorem $M$ is proven by a Zorn's lemma argument and in [2] Theorem K's proof uses the well-ordering theorem. We have shown that in Zermelo-Fraenkel set theory with only the axiom of choice for families of finite sets Theorems $\mathrm{H}, \mathrm{K}$, and $\mathrm{M}$ are equivalent. Thus these three technical results which have proven useful in obtaining local theorems are for all intents and purposes one and the same theorem. For those interested in the fine points of axiomatics, one can also show in the above mentioned version of set theory that the Boolean prime ideal theorem is equivalent to Theorems $\mathrm{H}, \mathrm{K}$, and $\mathrm{M}$. This is of interest since the Boolean prime ideal theorem is strictly weaker than the axiom of choice (see Halpern and Lévy [1]).

\section{BIBLIOGRAPHY}

1. J. D. Halpern and A. Lévy, The Boolean prime ideal theorem does not imply the axiom of choice, Proc. Sympos. Pure Math., vol. 13, part 1, Amer. Math. Soc., Providence, R.I., 1971, pp. 83-134.

2. A. G. Kuroš, Theory of groups. Vol. II, GITTL, Moscow, 1953; English transl., Chelsea, New York, 1960. MR 15, 501; MR 22 \#727. 
3. A. I. Malcev, On a general method for obtaining local theorems in group theory, Ivanov. Gos. Ped. Inst. Uč. Zap. Fiz.-Mat. Fak. 1 (1941), no. 1, 3-9. (Russian) MR $17,823$.

4. D. H. McLain, Local theorems in universal algebras, J. London Math. Soc. 34 (1959), 177-184. MR 21 \#3361.

5. A. Robinson, On the construction of models, Essays on the Foundations of Mathematics, Magnes Press, Hebrew Univ., Jerusalem, 1961, pp. 207-217. MR 29 \#23.

6. D. J. S. Robinson, Infinite soluble and nilpotent groups, Queen Mary College Math. Notes, Queen Mary College, London, 1967. MR 42 \#4635.

Department of Mathematics, Michigan State University, East lansing, MiCHIGAN 48823 